\title{
Inkjet printing of copper wire on PET substrate
}

\author{
E. Y. Wang ${ }^{1} \cdot$ Xuezhao $\mathrm{Li}^{1} \cdot$ Y. N. Duan ${ }^{1}$
}

Received: 15 April 2015/Accepted: 16 May 2015/Published online: 31 May 2015

(C) The Author(s) 2015. This article is published with open access at Springerlink.com

\begin{abstract}
A new process to fabricate copper pattern on PET substrate is developed in the experiment. Functional ink with ion-adsorption nanoparticles was printed on PET substrate to form the pattern adsorption film. Catalytic ion was adsorbed by amino groups in the adsorption film to catalyze the electroless plating process of copper. The mercapto groups were introduced to the film to enhance the reliability of patterns. In addition, the specific solvent was used in the ink to increase the surface roughness of adsorption film which leads to a better adhesion of the patterns. The conductivity of prepared copper patterns is the same as bulk copper and the patterns show good adhesion on PET substrate.
\end{abstract}

Keywords Copper patterns - Inkjet printing - PET substrate $\cdot$ Electroless plating $\cdot$ Ion adsorption

\section{Introduction}

Fabrication of conductive patterns by printed electronics has generated growing interest in recent years, and can be applied to the manufacture of printed circuit board (PCB), flexible printed circuit board (FPC) and integrated circuit (IC) (Kim et al. 2009; Gans and Duineveld 2004; Kang et al. 2010). Compared with the conventional lithographic and etching process, printed electronics process shows the advantages of less wastage of materials, less process steps, less pollution and less cost.

Xuezhao Li

lxztechnology@tom.com

1 Institute of Mechanical Engineering, University of Engineering Science, Shanghai, China
Inkjet printing of nanoparticles' conductive ink is widely researched in the additive fabrication of conductive circuits (Tai and Yang 2011; Reinhold et al. 2009). However, most of the conductive ink must be printed on heatresistant substrate in a high sintering temperature $\left(100-550{ }^{\circ} \mathrm{C}\right)$ by applying voltages or by laser curing. The conductivity of the prepared patterns is far below bulk metal. Besides, copper nanoparticles are easy to be oxidized; thus they must be prepared and sintered without oxygen. These problems increase the cost and limit the applications.

Printing of catalyst ink and metallization by electroless plating is a promising method. A few studies have reported inkjet printing of $\mathrm{Pd}$, which is often employed as a catalyst layer for the electrochemical deposition of other metals. Zabetakis et al. (2009) used a commercial Pd-Sn electroless catalyst, Cataposit 44 (Rohm \& Haas), to print a Pd catalyst on paper. Also, Busato et al. (2007) used ionic PdCl2 as the source of $\mathrm{Pd}$ and printed a pattern on polyimide. The patterns were baked at $608{ }^{\circ} \mathrm{C}$ for $15 \mathrm{~min}$. Reduction of the surface-bound $\mathrm{Pd}^{2+}$ to metallic Pd was accomplished by immersion into sodium borohydride at $20{ }^{\circ} \mathrm{C}$ for $5 \mathrm{~min}$. The printed patterns with $\mathrm{Pd}$ as a catalyst can be used as surface-enhanced Raman scattering substrates that exhibit high sensitivity (Bhuvana et al. 2010). This process can be applied on almost all kinds of substrates and does not need inert gas environment. Moreover, the conductivity can be the same with bulk metal. But the poor adhesion is the main problem. In order to guarantee the adhesion, the patterns must be very thin, thus the resistance is large. Surface modification of substrates can enhance the adhesion to a certain degree (Jeong et al. 2008). But the problem still exists when thick metal is deposited. Another potential method is 
based on selective adsorption of catalytic ion. Ion-adsorption polymer is coated or printed on substrates to form the pattern adsorption film, adsorption of the catalytic ion via complexation or electric charge. Then the patterns are metalized by electroless planting. However, ion-adsorption polymers, which contain amino, carboxyl, quaternary amine groups, etc., are always water-soluble (Dearden et al. 2005; Shah et al. 1999). The adsorption film will be swelled or dissolved in the electroless plating bath, causing the failure of the patterns. Besides, poor adhesion and large resistance problems still exist. Moreover, the use of palladium as catalyst increases the production cost.

In this paper, a new process based on selective adsorption of catalytic ion and electroless plating of copper to fabricate conductive patterns is developed. This method can be called patterning-adsorption-plating (PAP) process. Ion-adsorption nanoparticles were prepared by hydrolyzing 3-aminopropyltriethoxysilane (APTES) with 3-mercaptopropyltriethoxysilane (MPTES) together. Specific solvent was added to obtain the functional ink. Modified office inkjet printer was used to print the patterned adsorption film on substrate conveniently. Afterward, the substrate was immersed into silver nitrate solution. Silver ion can be adsorbed to the film via the complexation with amino groups. Finally, the patterns were metallized by electroless plating of copper with the catalyzing of silver. Sintering process can be avoided, so substrates with low heat distortion temperature (HDT) and PET (HDT $<90{ }^{\circ} \mathrm{C}$ ) can be used. The conductivity of the patterns can be the same as bulk copper. Mercapto groups are introduced to the nanoparticles to enhance the hydrophobicity and stability of the adsorption film. In order to improve the adhesion, we made use of the phenomenon in that the crystallization habits of nanoparticles differ in different solvents. The ionadsorption nanoparticles can crystallize in big particles from the solvent. It leads to higher surface roughness of the adsorption film and increases the adhesion of copper patterns.

\section{Experiment}

\section{Materials}

PET films, isopropanol, butanol, 2-methoxy ethanol, butyl acetate, MPTES (99\%), silver nitrate, copper sulfate, formaldehyde, potassium sodium tartrate, EDTA disodium, potassium ferrocyanide, sodium hydroxide were all AR grade purchased from Sinopharm Chemical Reagent Co., Ltd. APTSE (99 \%) and 2,2-bipyridyl was AR grade obtained from Shanghai Aladdin company.

\section{Preparation of functional ink}

APTES $(3 \mathrm{~g})$ and MPTES $(1 \mathrm{~g})$ were mixed in a sealed flask, and then ethanol $(0.4 \mathrm{~g})$, isopropanol $(0.1 \mathrm{~g})$ and water $(0.5 \mathrm{~g})$ were added with stirring at $25{ }^{\circ} \mathrm{C}$ for $24 \mathrm{~h}$. Afterward, different solvents $(20 \mathrm{~g})$ were added to produce the corresponding functional inks (Table 1).

\section{Fabrication of conductive patterns}

A modified office inkjet printer (Epson Mel+) was used to print the patterned adsorption film on PET substrate followed by drying in an oven at $70{ }^{\circ} \mathrm{C}$ for $30 \mathrm{~min}$. Then the substrate was put into silver nitrate solution at $50{ }^{\circ} \mathrm{C}$ for $5 \mathrm{~s}$, and rinsed by deionized water. Finally, the substrate was immersed into the copper electroless plating bath that comprised copper sulfate $(15 \mathrm{~g} / \mathrm{L}), 2,2$-bipyridyl $(0.02 \mathrm{~g} /$ $\mathrm{L})$, potassium ferrocyanide $(0.1 \mathrm{~g} / \mathrm{L})$ and formaldehyde $(15 \mathrm{ml} / \mathrm{L})$ at $40{ }^{\circ} \mathrm{C}$ for metallization. Additional heat treatment at $70{ }^{\circ} \mathrm{C}$ for $60 \mathrm{~min}$ can relieve the internal stress, leading to better adhesion and stability of the patterns.

\section{Characterization}

The Fourier transform infrared spectroscopy images were detected on Nicolet Nexus 470 FT-IR spectrometer. Contact angle was measured on Dataphysics OCA15+ optical contact angle measuring device. The surface profile was measured on Veeco Dektat 150 profile meter. The square resistance was obtained by a four-point probe. SEM (JEOL, JSM-6701F, Japan) was operated at $5 \mathrm{kV}$ to inspect the morphology (Fig. 1).

\section{Results and discussions}

\section{The stability of the adsorption film}

Ion-adsorption nanoparticles were prepared by hydrolyzing APTES and MPTES together. APTES and MPTES can react with water and generate polysiloxane nanoparticles containing hydroxyl, amino and mercapto groups (Fig. 2a). However, polysiloxane can also be hydrolyzed and broken down into small molecules, especially in alkaline solution (Fig. 2b). Meanwhile, the adhesion and stability of the adsorption film declined sharply. So, it is vital to prevent the adsorption film from hydrolyzing in alkaline electroless plating bath.

One solution is to increase the hydrophobicity of the adsorption film. The hydrophobic groups on the surface can block the water from penetrating into the interior. So the 
Table 1 The relationship between the adhesion of the conductive patterns and the solvent used in the functional ink

\begin{tabular}{lll}
\hline Solvent & Surface roughness $(\mu \mathrm{m})$ & Adhesion $^{\mathrm{a}}$ \\
\hline Butanol: 2-methoxy ethanol $=1: 2$ & 0.001 & $0 \mathrm{~B}$ \\
Butanol: 2-methoxy ethanol:butyl acetate $=1: 2: 1$ & 0.027 & $0 \mathrm{~B}$ \\
Butanol: 2-methoxy ethanol:butyl acetate $=1: 2: 3$ & 0.296 & $5 \mathrm{~B}$ \\
Butanol: 2-methoxy ethanol:butyl acetate $=1: 2: 6$ & 0.471 & $0 \mathrm{~B}$ \\
\hline
\end{tabular}

${ }^{a}$ The adhesion of the conductive patterns was measured following the standard ASTM D3359. 0B is the worst, 5B is the best. The electroless plating temperature is $40^{\circ} \mathrm{C}$ and the plating time is $15 \mathrm{~min}$

Fig. 1 The schematic diagrams of PAP process

Fig. 2 a Synthesis process of ion-adsorption nanoparticle. b Hydrolyzing of the ionadsorption in alkaline solution. c The contact angle of water on the adsorption film with mercapto groups. d The contact angle of water on the adsorption film with mercapto groups

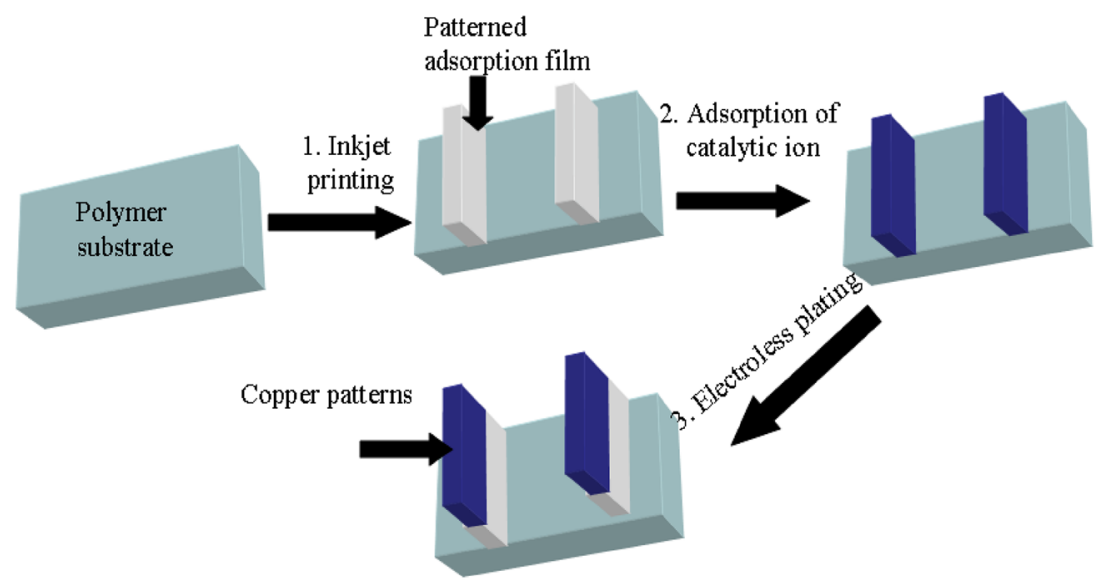

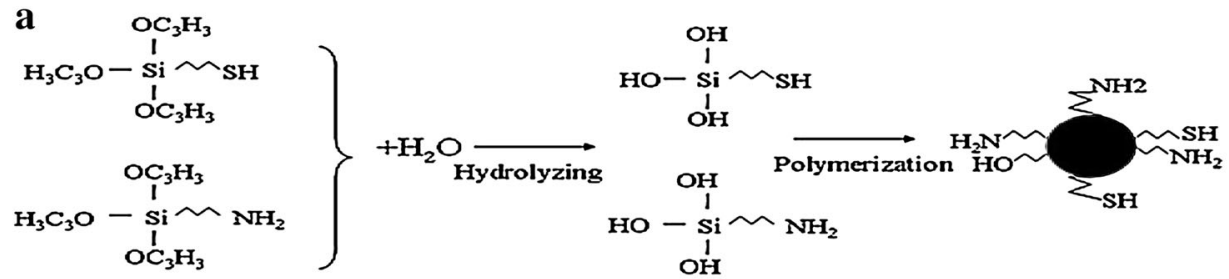

$\mathbf{b}$

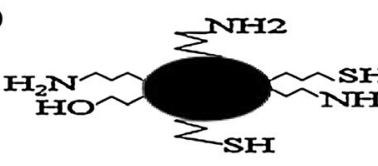

$+\mathrm{H}_{2} \mathrm{O}$
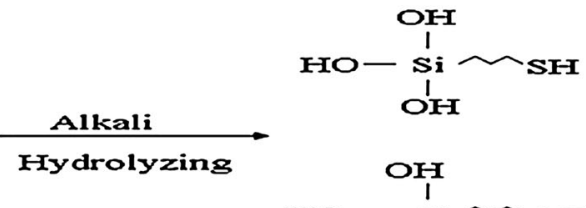

c

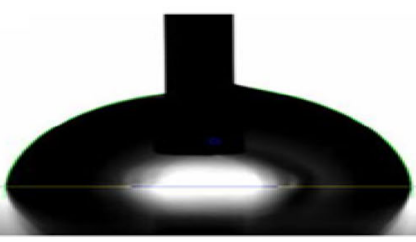

d

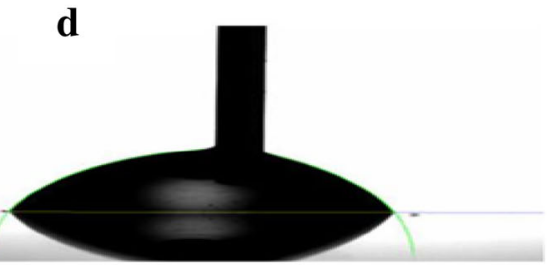

hydrolysis is slowed down, and the adsorption film can maintain stability for longer time. For comparison, the ionadsorption nanoparticles hydrolyzed only by APTES were prepared. After being printed on PET film, the adsorption film was immersed into the electroless planting bath at $40{ }^{\circ} \mathrm{C}$. However, the film was swelled and can be easily shelled off after $10 \mathrm{~min}$. In order to increase the hydrophobicity, mercapto group was introduced. The contact angle of water on the adsorption film with mercapto groups is $33.4^{\circ}$ (Fig. 2c). Compared to the film without mercapto groups $\left(19.4^{\circ}\right.$, Fig. 2d), the hydrophobicity increases greatly. The adsorption film was also immersed into the 
electroless plating bath; 30 min later, the film can still be stable and adhere firmly to the substrate. So, the hydrophobic adsorption film can be metalized for longer time, and the conductive patterns with lower resistance are thus obtained.

\section{The relationship between the solvent and the adhesion of the conductive patterns}

The interface of adsorption film and PET substrate is strong. The residual hydroxyl groups on the nanoparticles can form chemical bonds with carboxyl and hydroxyl groups on the PET substrate. Theoretically, the adsorbed silver ion is reduced to silver nanoparticles by formaldehyde by electroless planting. Mercapto groups can react with silver nanoparticles and form $\mathrm{Ag}-\mathrm{S}$ bond, thus the catalyst is tightly bonded to the adsorption film. The adhesion between the adsorption film and the deposited copper is related to the surface roughness of the substrates. The enhanced surface roughness leads to better adhesion in a certain range, for the film with coarse surface can mechanically anchor the deposited metal tightly. Adding filler particles to the ink can increase the surface roughness of the adsorption film. But the particles will block the nozzle seriously. So, a novel method was applied to fulfill the requirements of inkjet printing and high surface roughness. It is based on the different crystallization habits of nanoparticles.

Ion-adsorption nanoparticles tend to suspend in solvent with medium polarity, such as ethanol, 2-methoxy ethanol, butanol, etc. with low polarity, are the poor solvents of these nanoparticles. As solvent evaporates, nanoparticles start to crystallize. In the ink with good solvent, nanoparticles tend to separate out and assemble in a continuous structure (Fig. 3a). So the formed adsorption film is very smooth. In the ink with poor solvent, the ion-adsorption nanoparticles will congregate and precipitate in the form of big particles (Fig. 3b). Accordingly, the adsorption film is very coarse.

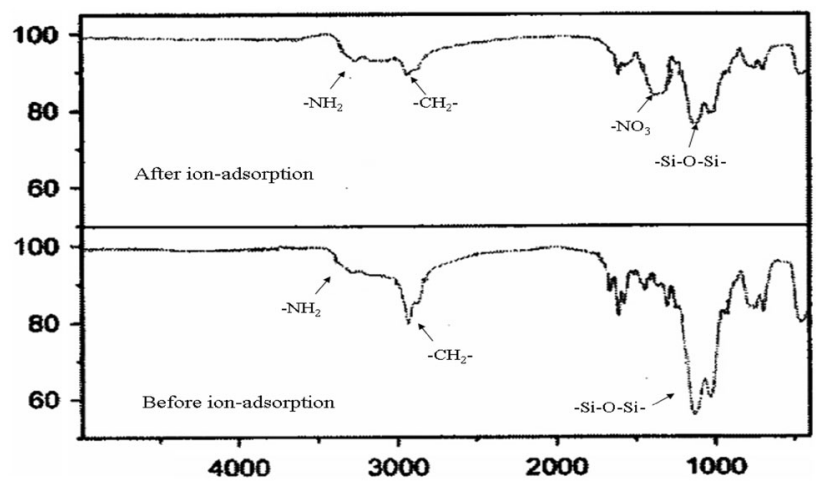

Fig. 4 The FTIR images of the adsorption film before and after adsorbing silver ion

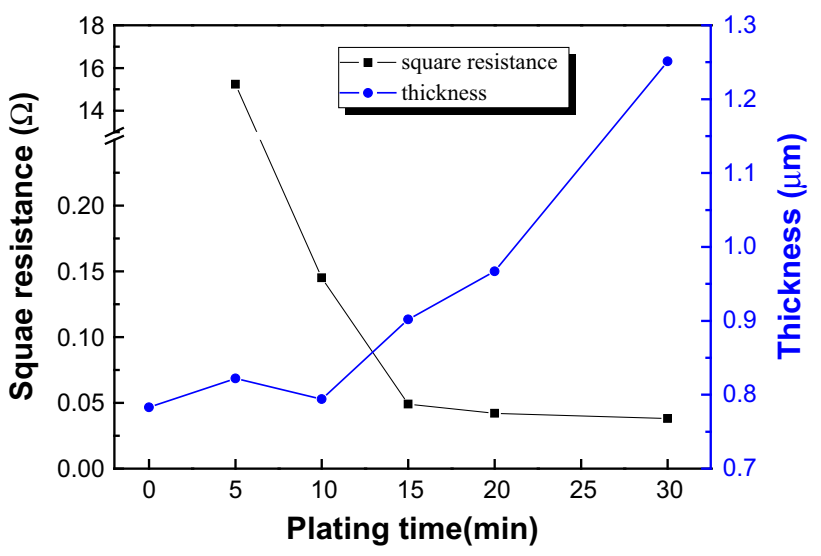

Fig. 5 The relationship between square resistance/thickness and plating time
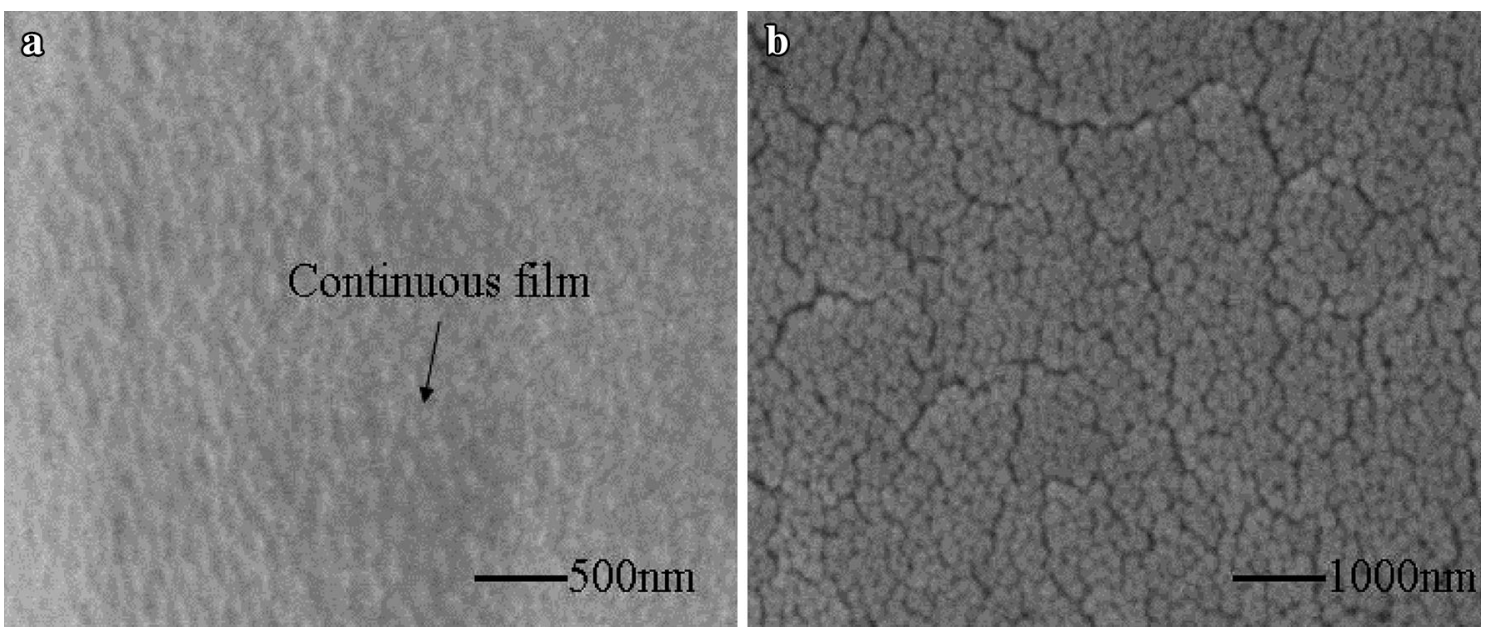

Fig. 3 SEM images of the film, a continuous film, b separated particles 
Fig. 6 The optical image (a) and the SEM image (b)

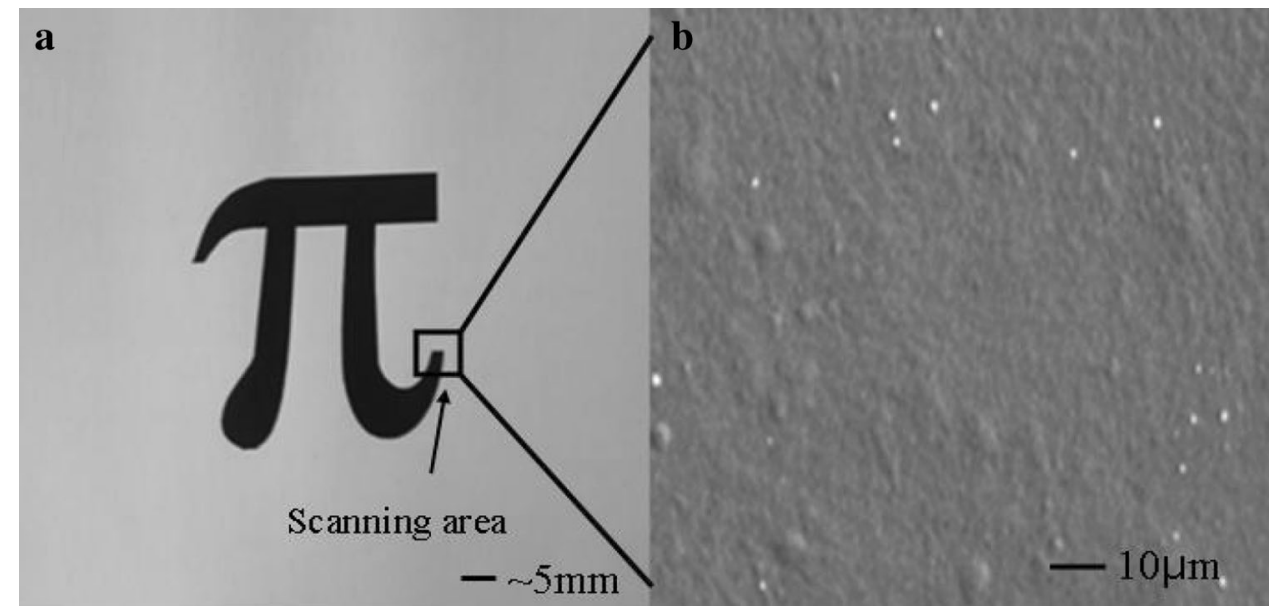

\section{Ion-adsorption}

Figure 4 demonstrates the FTIR of adsorption film. The main difference before and after ion-adsorption is the peak around $1372 \mathrm{~cm}^{-1}$, which is ascribed to the stretching vibration of $\mathrm{NO}_{3}{ }^{-}$. The adsorbed silver ion exists in the form of silver-amine ion. This electropositive ion can attract $\mathrm{NO}_{3}{ }^{-}$negative ion via Coulomb force. The bands at 3276, $3167 \mathrm{~cm}^{-1}$ (before ion-adsorption) and $3267,3161 \mathrm{~cm}^{-1}$ (after ion-adsorption) are assigned to the asymmetric and symmetric and symmetric stretching of $-\mathrm{NH}_{2}$. Silver ion can strengthen the stretching vibration of coordinated $\mathrm{NH}_{2}$. So the peaks after ion-adsorption are sharper.

\section{Properties of the conductive patterns}

The square resistance of the conductive patterns with different plating time is shown in Fig. 5. The square resistance falls sharply and reaches $0.145 \Omega$ at $10 \mathrm{~min}$. Afterward the square resistance goes down slowly to $0.0308 \Omega$ at $30 \mathrm{~min}$. The plating time and square resistance are in inverse proportion, agreeing well with theoretic calculation. The thickness of the copper patterns is also shown in Fig. 5a. Before electroless plating, the thickness was $0.783 \mu \mathrm{m}$. However, no obvious change is found before $10 \mathrm{~min}$, which means the copper is initially deposited inside the adsorption film. This phenomenon to some extent improves the adhesion of the deposited copper; $10 \mathrm{~min}$ later, copper starts to grow on the surface of adsorption film at a rate of $0.0216 \mu \mathrm{m} / \mathrm{min}$. The resistivity of the deposited copper is calculated to be $1.76 \times 10^{-6} \Omega \mathrm{cm}$, very close to cubic copper $\left(1.68 \times 10^{-6} \Omega \mathrm{cm}, 20^{\circ} \mathrm{C}\right.$. After conservation in a dry and enclosed environment for 3 months, the value remains unchanged.

The optical image of the prepared sample is shown in Fig. 6a. The resolution of the pattern is related to the method of patterning. The modified office inkjet printer can print the line as thin as $100 \mu \mathrm{m}$. Finer line can be achieved by a better printer. The SEM image (Fig. 6b) indicates that the conductive patterns are compact and continuous without void or crack. It can explain the excellent conductivity of the deposited copper.

\section{Conclusions}

A new PAP method to fabricate conductive patterns is developed. The main steps include inkjet printing of the ion-adsorption functional ink, ion-adsorption of catalytic ion and electroless plating of copper. A novel ion-adsorption nanoparticles' functional ink was prepared. Silver ion coordinates with the amino groups on the nanoparticles and is thus adsorbed to the substrate. Introduced Mercapto groups enhance the stability of the adsorption film. Mixed solvent was used in the functional ink to increase the surface roughness of the adsorption film, resulting in an improved adhesion of the deposited copper. The resistivity of the copper patterns is about the same with bulk copper, and the adhesion is also excellent. In general, this cost-effective and pollution-decreased process can, to some extent, substitute the traditional process, especially in the manufacture of FPC.

Open Access This article is distributed under the terms of the Creative Commons Attribution 4.0 International License (http:// creativecommons.org/licenses/by/4.0/), which permits unrestricted use, distribution, and reproduction in any medium, provided you give appropriate credit to the original author(s) and the source, provide a link to the Creative Commons license, and indicate if changes were made.

\section{References}

Bhuvana T, Boley W, Radha B et al (2010) Inkjet printing of palladium alkanethiolates for facile fabrication of metal 
interconnects and surface-enhanced Raman scattering substrates. Micro Nano Lett 5:296-299

Busato S, Belloli A, Ermanni P (2007) Inkjet printing of palladium catalyst patterns on polyimide film for electroless copper plating. Sens Actuat B Chem 123:840-846

Dearden AL, Smith PJ, Shin DY, Reis N, Derby B, O’Brien P (2005) A low curing temperature silver ink for use in ink-jet printing and subsequent production of conductive tracks. Macromol Rapid Commun 26(4):315-318

Gans BJ, Duineveld PC (2004) Inkjet printing of polymers: state of the art and future developments. Adv Mater 16:203-213

Jeong SH, Woo KH, Kim DJ et al (2008) Controlling the thickness of the surface oxide layer on $\mathrm{Cu}$ nanoparticles for the fabrication of conductive structures by ink-jet printing. Adv Funct Mater 18(5):679-686

Kang JS, Kim HS, Ryu J et al (2010) Inkjet printed electronics using copper nanoparticle ink. J Mater Sci 21(11):1213-1220
Kim D, Lee SH, Jeong S (2009) All-ink-jet printed flexible organic thin-film transistors on plastic substrates. Electrochem SolidState Lett 12:195-197

Reinhold I, Hendriks CE, Eckardt R et al (2009) Argon plasma sintering of inkjet printed silver tracks on polymer substrates. J Mater Chem 19(21):3384-3388

Shah P, Kevrekidis Y, Benziger J (1999) Ink-jet printing of catalyst patterns for electroless metal deposition. Langmuir 15(4):1584-1587

Tai YL, Yang ZG (2011) Fabrication of paper-based conductive patterns for flexible electronics by directwriting. J Mater Chem 21(16):5938-5943

Zabetakis D, Loschialpo P, Smith D (2009) Direct-write patterning palladium colloids as a catalyst for electroless metallization for microwave composites. Langmuir 25:1785-1789 